\title{
Is irrational use of tranexamic acid justified in anesthesia practice?
}

\author{
Bhavna Gupta \\ Department of Anesthesiology, Maulana Azad Medical College and Lok Nayak Hospital, New Delhi, India
}

Tranexamic acid (TA) is a synthetic derivative of the amino acid lysine, which binds to five lysine binding sites on the plasminogen molecule. It has been widely used in orthopedic, cardiac, gynecological, and neurosurgery, etc., to reduce bleeding and transfusion requirements. The intravenous dosage is typically $0.5-1 \mathrm{~g}$, administered by slow injection three times per day. Alternatively, the initial dose can be followed by infusion of $25-50 \mathrm{mg} / \mathrm{kg}$ over 24 hours. Dosing should be reduced to 5-10 $\mathrm{mg} / \mathrm{kg}$ in patients with renal failure. TA has also proven useful in emergency trauma victims to reduce bleeding, as demonstrated in the CRASH2 trial, in which patients at risk of bleeding given a loading dose of $1 \mathrm{~g}$ of TA over 10 minutes, followed by infusion of $1 \mathrm{~g}$ over 8 hours within 8 hours of trauma, were found to have less bleeding compared to those receiving placebo [1]. Although there is no consensus regarding the adequate dose of TA to prevent bleeding in various surgeries, there have been many reports highlighting the dosages required for the optimal effect. Sigaut et al. [2] used two doses of TA, $30 \mathrm{mg} / \mathrm{kg}$ and $10 \mathrm{mg} / \mathrm{kg}$, and reported that the higher dose was more effective for reducing transfusion needs, blood loss, and the incidence of repeat surgery.

There are various contraindications for the use of TA: in cases of active thromboembolic disease, e.g., deep venous thrombosis or pulmonary embolism, TA has been shown to have a procoagulant effect and can be unsafe in patients with thromboembolism, those in a hypercoagulable state, and those

Corresponding author: Bhavna Gupta, M.D.

Department of Anesthesiology, Maulana Azad Medical College and Lok Nayak Hospital, New Delhi 110059, India

Tel: 91-8527686660, Fax: 91-1126853108

Email: bhavna.kakkar@gmail.com

ORCID: https://orcid.org/0000-0002-3108-0408

Received: August 28, 2017.

Revised: September 18, 2017.

Accepted: September 27, 2017.

Korean J Anesthesiol 2018 June 70(3): 237-238

https://doi.org/10.4097/kja.d.18.27204 with thrombogenic vascular diseases. Sundström et al. [3] suggested that TA was associated with an increased risk of venous thromboembolism, although the risk estimate did not reach statistical significance. Hypersensitivity reactions to TA are not uncommon, and care should be taken during its slow, intravenous administration. Visual effects are known side effects of TA, and retinal arterial and venous occlusion have even been reported in the literature. When administered at a high dosage, TA is known to produce seizure in cases of cardiac surgery, where it is commonly used at a high dose, to ensure an effective concentration, secondary to gamma-aminobutyric acid (GABA) receptor inhibition. Murkin et al. [4] suggested the epileptogenic potential of TA at higher doses in susceptible individuals. They suggested that nonischemic seizure is associated with TA at doses of 61$259 \mathrm{mg} / \mathrm{kg}$ [4]. After this review, dosing guidelines have been modified to a low dose of tranexamic acid as $30 \mathrm{mg} / \mathrm{kg}$ loading dose followed by $15 \mathrm{mg} / \mathrm{kg} / \mathrm{h}$ as infusion [4]. Many authors have suggested that use of TA at doses exceeding $80 \mathrm{mg} / \mathrm{kg}$ should be avoided. However, one of the largest retrospective data analyses, of 4,883 cardiac surgical patients, indicated that even a moderate TA dose of $24 \mathrm{mg} / \mathrm{kg}$ was associated with almost double the rate of convulsions and in-hospital mortality in comparison to a reference group that did not receive the drug [5].

Extreme caution is required regarding the use of this drug in patients with disseminated intravascular coagulation requiring antifibrinolytic therapy. Even among patients with acute ureteral obstruction, clot formation has been reported in those with upper urinary tract bleeding. Concurrent administration of TA with oral contraceptives is not recommended, as the risk of thromboembolism is further increased. Concurrent administration with factor IX complexes or anti-inhibitor coagulant complexes is also not recommended, due to the risk of thrombosis. Cerebral infarction and edema have been reported in patients with subarachnoid hemorrhage receiving TA. Concurrent use of tretinoin with TA may exacerbate procoagulant effects. Accidental intrathecal injection of $500 \mathrm{mg}$ of TA was reported to be associated with spinal myoclonus, and even death. TA is

(c) This is an open-access article distributed under the terms of the Creative Commons Attribution Non-Commercial License (http://creativecommons.org/ licenses/by-nc/4.0/), which permits unrestricted non-commercial use, distribution, and reproduction in any medium, provided the original work is properly cited. 
labeled a category B drug in pregnancy, as it is well known to be excreted in breast milk. Fibrinolytic activity is very high in neonates, however, there is little evidence of any deleterious effect of suppressing it by TA in the first hours of life.

There are strong evidences regarding the relation between use of TA and reduction of bleeding during the perioperative period. However, the drug doge-effect relationship of TA in different surgeries, as well as its effect on thromboembolic events, seizure potential, morbidity, and mortality remain uncertain. For safe usage, anesthesiologists should be familiar with the mechanism of action of TA and its known side effects (nausea, vomiting, chills, fever, headache, muscle pain, stuffy nose, giddiness, dizziness, thromboembolic events, ocular effects, and hypotension on rapid intravenous injection). Most studies performed to date have examined the efficacy of TA after excluding patients with contraindications for its use, as well as those with a high-risk profile. Therefore, it is necessary for clinicians to assess the thromboembolic and seizure risks on an individual basis before administering TA. This drug should not be used in all surgeries, but rather its role should be well defined depending upon the patient's profile, and the duration and type of surgery.

\section{Acknowledgments}

I would like to acknowledge Dr. Anish Gupta, my husband for suggesting inputs related to the topic.

\section{References}

1. CRASH-2 trial collaborators, Shakur H, Roberts I, Bautista R, Caballero J, Coats T, et al. Effects of tranexamic acid on death, vascular occlusive events, and blood transfusion in trauma patients with significant haemorrhage (CRASH-2): a randomised, placebo-controlled trial. Lancet 2010; 376: 23-32.

2. Sigaut S, Tremey B, Ouattara A, Couturier R, Taberlet C, Grassin-Delyle S, et al. Comparison of two doses of tranexamic acid in adults undergoing cardiac surgery with cardiopulmonary bypass. Anesthesiology 2014; 120: 590-600.

3. Sundström A, Seaman H, Kieler H, Alfredsson L. The risk of venous thromboembolism associated with the use of tranexamic acid and other drugs used to treat menorrhagia: a case-control study using the General Practice Research Database. BJOG 2009; 116: 91-7.

4. Murkin JM, Falter F, Granton J, Young B, Burt C, Chu M. High-dose tranexamic acid is associated with nonischemic clinical seizures in cardiac surgical patients. Anesth Analg 2010; 110: 350-3.

5. Koster A, Börgermann J, Zittermann A, Lueth JU, Gillis-Januszewski T, Schirmer U. Moderate dosage of tranexamic acid during cardiac surgery with cardiopulmonary bypass and convulsive seizures: incidence and clinical outcome. Br J Anaesth 2013; 110: 34-40. 